\title{
Criminologie
}

\section{Éditorial : les droits des détenu-e-s devant les tribunaux}

\section{Lucie Lemonde}

Volume 24, numéro 1, 1991

Les droits des détenu-e-s

URI : https://id.erudit.org/iderudit/017299ar

DOI : https://doi.org/10.7202/017299ar

Aller au sommaire du numéro

Éditeur(s)

Les Presses de l'Université de Montréal

ISSN

0316-0041 (imprimé)

1492-1367 (numérique)

Découvrir la revue

Citer ce document

Lemonde, L. (1991). Éditorial : les droits des détenu-e-s devant les tribunaux.

Criminologie, 24(1), 5-9. https://doi.org/10.7202/017299ar d'utilisation que vous pouvez consulter en ligne.

https://apropos.erudit.org/fr/usagers/politique-dutilisation/ 
La question des droits des détenu-e-s a toujours soulevé controverses et débats passionnés. La lecture de ce numéro thématique montre bien que tel est encore le cas au début des années 1990 . Il est vrai que les conditions physiques de détention se sont améliorées depuis le premier pénitencier ${ }^{1}$, que le chat-à-neuf-queues a maintenant sa place au musée et que l'on ne décrit plus les prisonniers comme des esclaves de l' «État ${ }^{2} »$. Il n'en demeure pas moins toutefois que la prison, qui, pour employer les termes de Foucault, «quadrille au plus près le temps, l'espace et les mouvements ${ }^{3}$ » des hommes et des femmes qui y sont confinés, est une institution totale où la règle de droit est perçue comme une contrainte et une entrave à l'exercice du pouvoir discrétionnaire.

Au cours des années 1980 , le mouvement pour les droits des détenu-e-s s'est déplacé et, à l'instar de ce qui s'est passé aux États-Unis, il a mené ses revendications devant les tribunaux. La lutte juridique des détenu-e-s pour la reconnaissance de leurs droits a porté bien sûr sur l'exercice de droits fondamentaux, tel le droit de vote, qui, comme le démontrent Bernheim et Millette, a rencontré et rencontre toujours une grande résistance, mais surtout sur le droit d'être traité équitablement. En plus de contrôler l'étendue de la liberté des détenu-e-s pendant l'incarcération en déterminant l'endroit de la détention, les autorités carcérales contrôlent également la longueur de l'incarcération par le jeu des remises de peine et des libérations conditionnelles. Les détenu-e-s réclament que ces décisions qui les privent de liberté soient équitables, non arbitraires. Ils revendiquent également le droit de participer aux décisions affectant leur vie.

La règle de droit n'existe pas que pour le plaisir de juristes tatillons. L'équité et l'impartialité sont l'essence de la liberté. Tous ont à gagner d'un système juste. C'est ce que reconnait le Groupe de travail de la révision du droit correctionnel quand il affirme:

L'absence d'un système équitable qui protège les droits des détenu-e-s accroît les tensions de la vie carcérale. Une personne incarcérée ne peut être qu'indignée de se trouver dans une société fermée, régie non pas par la loi, mais par un pouvoir discrétionnaire qui peut s'avérer largement

1. Voir l'article de Jacques Laplante.

2. Ruffin c. Commonwealth, 62 Va. 790 (1871).

3. Michel Foucault, Surveiller et punir, Paris, Gallimard, 1975, p. 139. 
arbitraire. La tension qui en résulterait pourrait créer un climat de méfiance, pouvant mener à la violence et contraire aux intérêts non seulement des détenu-e-s, mais du personnel, de la direction et de la communauté en général ${ }^{4}$.

Ce même groupe de travail affirme cependant du même souffle que «la règle de droit demeure une contrainte importante dans le fonctionnement du système correctionnel ${ }^{5} »$. Est-ce à dire que le respect de la règle de droit signifie la perte de pouvoir et de contrôle?

Les tribunaux canadiens ont longtemps refusé d'assumer un rôle de surveillance des décisions internes du monde carcéral. Michael Jackson en était venu à la conclusion, en 1974, que le système correctionnel était un «État sans droit» perpétué par la grande réticence des tribunaux à intervenir derrière les murs de la prison ${ }^{6}$. Les conséquences de cette attitude de "laisser-faire ${ }^{7}$ " étaient que les détenu-e-s étaient abandonné-e-s à l'arbitraire des autorités, sans aucun forum judiciaire pour contester les abus de l'administration ${ }^{8}$.

Le principal motif de cette attitude de «laisser-faire» reposait sur la conception, encore tenace aujourd'hui, que la personne condamnée à l'emprisonnement perd inévitablement tous ses droits et ne jouit que de certains privilèges discrétionnaires. La mort civile a été abolie au Canada en 1892 mais les détenu-e-s ont continué à être perçu-e-s comme des personnes déchues de leurs droits. Plus récemment, dans un virulent jugement dissident, le juge Brennan de la Cour suprême américaine exprimait bien le sentiment de rejet social dont sont victimes les détenu-e-s:

Il est tentant de penser que les prisonniers font partie d'un autre monde mais cela ne change rien au fait que cette société qu'ils habitent est la nôtre. Quand un prisonnier émerge de l'ombre pour demander un redressement constitutionnel, il n'invoque pas une série de principes étranges venus d'une lointaine culture. Il parle le langage de la Charte et nous devons l'écouter. Il nous demande de reconnaître que le pouvoir exercé

4. A. MacPhail, G. Parry, D. Weir, R. Cormier et H. Bebbington, Cadre pour la révision du droit correctionnel, Document de travail $n^{\circ} 2$, Ottawa, Solliciteur général du Canada, 1986, p. 39.

5. Ibid., La Philosophie correctionnelle, Document de travail $n^{\circ}$ 1, p. 27.

6. Michael Jackson, «The Right to Counsel in Prison Disciplinary Hearings» (1986), University of British Columbia Law Review, vol. 20, p. 221; M. Jackson, «Justice Behind the Walls. A Study of the Disciplinary Process in Canadian Penitentiary» (1974), Osgoode Hall Law Journal, vol. 12, p. 1.

7. Ce que les Américains ont appelé le hands off. Pour une analyse critique de cette doctrine, voir «Beyond the Ken of the Courts: A Critique of Judicial Refusal to Review the Complaints of Convicts» (1962-1963), Yale Law Journal, vol. 72, p. 506.

8. Pour un historique complet de l'intervention judiciaire en droit carcéral, voir Lucie Lemonde, L'Habeas corpus en droit carcéral, Cowansville, Éd. Yvon Blais, 1990, $130 \mathrm{p}$. 
dans l'ombre doit l'être dans des limites raisonnables'. (C'est nous qui traduisons.)

La reconnaissance par la Cour suprême du Canada de l'existence de droits pour les détenu-e-s, de même que la reconnaissance de l'obligation pour les autorités carcérales d'agir équitablement à leur endroit, n'est survenue qu'en 1980. Tout d'abord, la Cour suprême a clairement énoncé qu' «une personne emprisonnée conserve tous ses droits civils autres que ceux dont elle a été expressément ou implicitement privée par la loi ${ }^{10}$ ». Ensuite, elle a précisé : "Le principe de la légalité doit régner à l'intérieur des murs d'un pénitencier ${ }^{11 . " ~}$

Suite à ces deux affaires, a commencé une ère d'activisme judiciaire. Plusieurs décisions touchant tous les aspects de la vie carcérale, discipline, ségrégation, transfèrement, conditions de détention, fouille, remise de peine, libération conditionnelle, ont été contestées avec succès devant les tribunaux. Le groupe de travail du Solliciteur général reconnaît que «l'intervention judiciaire a joué, et continue de jouer, un rôle de premier plan ${ }^{12}$ » dans la promotion des droits des détenu-e-s mais, selon lui, «le Parlement est mieux placé pour traiter ces questions, dans le cadre d'une révision fondamentale du droit correctionnel, que ne le sont les tribunaux, qui les traitent cas par cas ${ }^{13}$ ».

Lorsque la Cour suprême affirme que la règle de droit doit régner à l'intérieur des murs de la prison, elle ne fait pas du «cas par cas». C'est souvent l'administration qui refuse d'étendre à tous une décision favorable à un détenu et d'appliquer de façon uniforme et généralisée les énoncés de principe des tribunaux. L'affaire Le Bar, pour ne prendre qu'un exemple, est très éloquente à cet égard. La Cour d'appel fédérale avait, le 19 juillet 1982, rendu une décision sur la façon de calculer la sentence dans l'affaire MacIntyre. L'avocat de LeBar, le 13 août suivant, avisait les autorités du pénitencier de l'effet de ce jugement sur le calcul de sentence de son client qui aurait dû être libéré le 11 août. Le Bar n'a été libéré que le 22 septembre. Dans une action en dommages- intérêts pour ces 43 jours de détention illégale, la Couronne a plaidé que le jugement de la Cour d'appel n'avait pas force de chose jugée et ne s'appliquait pas dans les cas similaires. Le juge s'est insurgé contre cette assertion et contre le manquement d'appliquer le droit aux personnes dans la même situation. «De ce retard énorme et inexpliqué, a-t-il dit, on ne peut

9. O'Lone v. Estate of Shabazz, 107 S.Ct. 2400, 2407 (1987).

10. Solosky c. La Reine [1980], 1 R.C.S. 823, 839. 602,622 .

11. Martineau c. Comité de discipline de l'institution de Matsqui, [1980] 2 R.C.S.

12. Loc. cit., note 4, p. 65.

13. Ibid., p. 30 . 
que conclure à la négligence et au mépris intentionnel et injustifié du droit du demandeur à la liberté ${ }^{14}$.»

Il existe un monde entre l'affirmation judiciaire d'un droit et son application quotidienne. La réaction du gouvernement à la reconnaissance du droit à la représentation par avocat devant les tribunaux disciplinaires des pénitenciers a consisté à tenter par tous les moyens de contourner la décision de la Cour d'appel fédérale dans l'affaire Howard c. Le tribunal disciplinaire de Stoney Mountain ${ }^{15}$ afin que les détenu-e-s ne puissent exercer ce droit. Dans Howard, la Cour a décidé que le droit à l'avocat aurait dû être accordé à cause de la gravité de la sanction susceptible d'être imposée, soit la perte de la remise de peine que le détenu avait à son crédit.

En réaction directe au jugement Howard, le gouvernement a créé une nouvelle catégorie d'infractions dites intermédiaires pour lesquelles il ne peut y avoir de sanction de perte de bon temps. On pensait ainsi éviter le jugement Howard. Le raisonnement est simple: pas de perte de bon temps, donc pas de conséquences graves, donc pas d'avocat. De plus, Michael Jackson, dans son article «The Right to Counsel in Prison Disciplinary Hearings ${ }^{16} »$, rapporte qu'une note de service a été remise à tous les directeurs leur enjoignant de ne plus porter d'accusations majeures sauf dans les cas d'assaut ou de voies de faits graves, mais uniquement des accusations intermédiaires, de sorte qu'il n'y ait jamais d'avocat ${ }^{17}$.

Il est compréhensible que l'on ait opposé une résistance à l'exercice de ce droit, car la reconnaissance du droit à l'avocat devant les tribunaux disciplinaires constitue une brèche dans l'institution totale du fait qu'elle implique la présence de témoins extérieurs de cette justice quelque peu expéditive ${ }^{18}$. D'ailleurs, ne se contentant plus de se prononcer sur le respect de l'équité procédurale, les tribunaux ont commencé à casser des décisions disciplinaires pour cause d'absence de preuves ${ }^{19}$.

14. LeBar c. La Reine, [1987] 1 C.F. 5895, confirmé à (1988) 46 C.C.C.(3d) 103.

15. Howard c. Président du tribunal disciplinaire de Stoney Mountain, [1984] 2 C.F 642; permission d'appeler accordée, puis appel jugé théorique, [1987] 2 R.C.S. 68.

16. Loc. cit., note 6, p. 278.

17. Depuis, les tribunaux ont affurmé que ce n'était pas la classification de l'infraction qui est déterminante mais bien la gravité de la sanction et que la condamnation à l'isolement cellulaire, «le trou», était grave car privative de liberté. Voir, entre autres, Tremblay c. Président du Tribunal disciplinaire de Laval, [1986] 25 Adm.L.R. 235.

18. Une enquête menée par l'Office des droits des détenu-e-s auprès du directeur de la prison de Bordeaux montre qu'en 1982, il y a eu 3561 comparutions devant le tribunal disciplinaire de cette institution qui ont donné lieu à 3561 verdicts de culpabilité. Les résultats de cette enquête non publiée sont déposés au secrétariat de la revue Criminologie.

19. Voir, entre autres, Gosselin c. Bouwman, C.F, T-587-85, 23 janvier 1986, j. Strayer; Clark c. Fox, C.F., T-1128-88, 24 juin 1988, j. Collier. 
Il y a eu, devant les tribunaux, des gains importants et significatifs. Des études américaines ${ }^{20}$ ont démontré que l'activité légale des prisonniers avait des effets positifs en améliorant leur attitude à l'égard du système et leur conduite en institution. Il semble également que cette activité légale réduise la tension, l'anxiété et l'hostilité entre les détenu-e-s et, finalement, serve la société tout entière au sein de laquelle tôt ou tard ces détenu-e-s retourneront.

Mais il est évident qu'à lui seul le recours judiciaire est insuffisant pour operer des changements réels et réduire de façon importante l'arbitraire. Les ordonnances des tribunaux ne sont pas toujours appliquées uniformément et, règle générale, elles n'ont pas encore franchi les portes des centres d'accueil pour jeunes. D'autres problèmes ont surgi, auxquels il faudra porter une attention particulière au cours des prochaines années. On parle beaucoup aujourd'hui de surpopulation, de surreprésentation des autochtones, de suréloignement des nouvelles constructions, etc. Chez nos voisins du sud, on dit ironiquement que la seule institution sociale américaine contrôlée par les Noirs est la prison ${ }^{21}$. La situation canadienne n'est pas aussi critique, mais nous ne sommes pas à l'abri d'un tel développement.

Les textes qui suivent sont autant d'éléments de réflexion et d'analyse sur le thème des droits des détenu-e-s et sur la vie carcérale. Les contributions sont variées, multidisciplinaires et, à l'image de la polémique entourant ce thème, le dossier se termine par deux visions sur les conclusions à tirer de vingt ans de discours et d'action en matière de droits des détenu-e-s.

20. Geoffrey P. Alpert, J. Finney et J. F Short, «Legal Services, Prisoners' Attitudes and "Rehabilitation", Journal of Criminal Law and Criminology, vol. 69, pp. 616-626 (1978); G. Alpert, Legal Rights of Prisoners: An Analysis of Legal Aid, Lexington, D.C. Heath, 1978 .

21. James J. Jacobs, «Race Relations and the Prison Subculture», in N. Morris et M. Tonry (éd.), Crime and Justice: An Annual Review of Research, vol. 1, Chicago, University of Chicago Press, 1979, p. 24. 